\title{
Técnicas y estrategias para desarrollar el turismo experiencial en humedales
}

\author{
Ma José Viñals Blasco \\ mvinals@cgf.upv.es \\ Pau Alonso-Monasterio \\ ambpau@gmail.com \\ Universidad Politécnica de Valencia, España
}

\section{RESUMEN}

Se han llevado a cabo numerosos esfuerzos en materia de Comunicación, Educación, Concienciación y Participación (CECoP) desde diversas administraciones y organizaciones conservacionistas en relación a la conservación de los humedales, pero los resultados han sido discretos si se comparan con los obtenidos en otros ecosistemas. Los humedales no gozan todavía del aprecio de la mayoría del público generalista, aunque son destinos habituales para ecoturistas especializados (p.e. observadores de aves).

Este trabajo identifica y analiza, por una parte, las causas de esta situación como son la complejidad de evidenciar los atractivos naturales de los humedales y la existencia de una percepción negativa de estos ecosistemas entre ciertos sectores del público.

Por otro lado, se presentan estrategias para abordar estas cuestiones y establecer una conexión más personal entre el público y los recursos que facilite el desarrollo de emociones en los visitantes. Así, se propone: un conocimiento en profundidad de los diferentes públicos, la identificación de nuevos iconos interpretativos, la puesta en valor del patrimonio intangible de los humedales, entre otros. Se describen también técnicas para reforzar la percepción sensorial de los visitantes in situ, ya que de ello depende, en gran medida, la satisfacción de la experiencia que, a su vez, está en la base de futuras actitudes y comportamientos proactivos de los visitantes en relación a la conservación, salvaguarda y disfrute de los humedales.

Palabras clave: Humedales, Experiencia turística, Emociones, Interpretación del Patrimonio. 


\title{
Techniques and strategies to develop Experiential Tourism in Wetlands
}

\begin{abstract}
Numerous Communication, Education, Participation and Awareness (CEPA) efforts have been carried out by different authorities and conservation organizations in relation to the conservation of wetlands. However, the results are very modest compared to those obtained in other ecosystems. Wetlands are not yet appreciated by most of the general public, although they are common destinations for specialized eco-tourists (e.g. birdwatchers).

This paper identifies and analyses the causes of this situation, which include the complexity of evidencing the wetlands' natural attractions and the existence of a negative perception of these ecosystems among certain sectors of the public.

In addition, strategies are presented to address these issues and to establish a more personal connection between the public and heritage resources in order to appeal to the emotions of visitors. Therefore the following actions are proposed: acquiring an in-depth knowledge of the different audiences, identifying new wetland interpretive wildlife icons, enhancing the intangible heritage of wetlands, among others. The paper also describes techniques to positively influence visitors' on-site sensorial perception as this influences the satisfaction with the experience. This is because a good experience forms the basis of future attitudes and proactive behaviours in favour of conservation, preservation and the enjoyment of wetlands.
\end{abstract}

Key Words: Wetlands, Tourism Experience, Emotions, Heritage Interpretation.

\section{INTRODUCCIÓN}

Los humedales, a diferencia de otros ecosistemas, detentan una consideración social que incluye sentimientos contradictorios que se plasman, por un lado, en un elevado reconocimiento ecológico debido a la biodiversidad que albergan, pero a la vez, se constata a nivel mundial una continuada pérdida de superficie de estos ecosistemas. Desde círculos científicos, el valor de estos ecosistemas ha sido siempre reconocido y es a partir de la década de los años '70 del pasado siglo cuando las administraciones comienzan a proteger al más alto nivel gran parte de los humedales mundiales, coincidiendo además con la puesta en marcha de la Convención sobre Humedales de Importancia Internacional en Ramsar (Irán) en 1971. Se trata pues del único ecosistema al que se le ha dedicado un tratado internacional para promover su protección. Si partimos de esta base, cabe preguntarse ¿por qué todavía son pues los ecosistemas más perseguidos y los que desaparecen a mayor velocidad?, ¿por qué los humedales no han recabado un respaldo mayoritario del público que impida que estas transgresiones se produzcan continuamente en todo del mundo?

Estas preguntas se hacen evidentes si consideramos que los humedales detentan importantes valores educacionales y se pueden considerar como "laboratorios vivos" idóneos para la formación especialmente en las ciencias naturales (Ecología, Biología, Geología, Hidrología, etc.) y también en tradiciones y formas de vida locales. Se trata, en definitiva, de extraordinarios lugares para la práctica de actividades recreativas interpretativas. Basados en estos argumentos, en muchos países se han dedicado 
numerosos esfuerzos a programas de Comunicación, Educación, Concienciación y Participación (CECOP) impulsados desde la Convención de Ramsar $^{1}$ en relación a la conservación de los humedales, pero la impresión que se tiene en el ámbito de los gestores de humedales, sin poder constatar este hecho con cifras, es que los resultados han sido discretos si se comparan con los obtenidos en otros ecosistemas. No obstante, los resultados de todas esas actividades de sensibilización han contribuido notablemente a la educación de grupos de escolares que han visitado regularmente los humedales como parte de sus actividades formativas.

Hay que señalar además, que los humedales se encuentran entre los ecosistemas de mayor biodiversidad del planeta. Detentan importantes valores faunísticos y florísticos considerados como sus principales atributos, y también funciones tangibles e intangibles entendidas en términos de ecoservicios (Bernués et al., 2001). Pues bien, muchas de estas funciones al ser intangibles son difíciles de percibir, transmitir y ser apreciadas por la sociedad. Respecto a la fauna hay que destacar que los principales grupos faunísticos que habitan los humedales son insectos, reptiles, anfibios, peces y aves. Todos estos grupos taxonómicos presentan alguna dificultad intrínseca para constituirse como un atractivo innato para el público generalista, salvo las aves. Los primeros, porque desde el punto de vista de su valoración recreativa se catalogan incluso como fauna molesta, los anfibios y reptiles porque no se encuentran entre los animales más atractivos para el gran público (Martin, 1997; Tremblay, 2002) y los peces porque no son fáciles de observar. Sin embargo, las aves son el gran atractivo de los humedales y hay que señalar que en torno a este recurso faunístico sí se ha generado siempre un importante flujo de ecoturistas muy bien identificado (observadores de aves, avituristas o birdwatchers).

Respecto a la percepción negativa que el público tiene de los humedales se debe a prejuicios que, en parte, están alimentados por una malentendida tradición cultural que se arrastra desde siglos precedentes y que tiene que ver con la idea de insalubridad, intransitabilidad, improductividad, etc. con que eran percibidos estos ecosistemas desde antaño. Los medios de comunicación y la cinematografía tampoco han ayudado a cambiar esta idea ya que siempre han abundado sobre su imagen más tenebrosa.

Revertir esta percepción negativa es el primer paso para conseguir un cambio de actitud de los visitantes hacia un mayor aprecio de estos ecosistemas que garantice comportamientos futuros de protección y salvaguarda. Las autoras de este trabajo entienden que todo este proceso basa su éxito en la provisión de una experiencia satisfactoria al visitante durante su periplo por los humedales que se apoya en la dimensión emocional de la visita.

\section{OBJETIVOS Y METODOLOGÍA}

A pesar de todos los esfuerzos para concienciar sobre el valor de los humedales tal como hemos visto, se puede decir que no gozan todavía del aprecio de la mayoría del público generalista. Por ello, este trabajo se plantea como objetivo general indagar sobre las causas que han desembocado en esta situación y también el de proponer algunas estrategias

\footnotetext{
${ }^{1}$ http://www.ramsar.org/es/actividad/el-programa-de-cecop-de-ramsar
} 
para revertir esa percepción negativa que todavía existe en ciertos sectores del público acerca de los humedales.

Este estudio se basa en un enfoque teórico y se ha llevado a cabo a partir del conocimiento y la experiencia académica y profesional desarrollada sobre los aspectos referidos a la conservación y uso público en humedales durante numerosos años de dedicación al abordaje de los mismos desde diferentes perspectivas y en diversos ámbitos geográficos: estudios hidromorfológicos y geoestratigráficos, desarrollos cartográficos, determinación de valores y funciones, inventario y valoración de recursos, redacción de planes de ordenación de los recursos naturales y planes de gestión, gestión de visitantes, procesos de participación, diseño de equipamientos y rutas interpretativas, identificación de impactos, estudios sobre turismo en humedales, diseño y desarrollo de herramientas de gestión del uso público, asesoramiento técnico a la Convención de Ramsar y a la iniciativa MedWet, etc.

Todo este bagaje académico y profesional, que ha sido divulgado en diversas publicaciones, se ha combinado con la revisión de trabajos académicos que han sido referidos a lo largo del documento y que versan fundamentalmente sobre la dimensión emocional de la interpretación. Ello ha dado lugar a un trabajo original que aplica estas nuevas tendencias en la interpretación del patrimonio a un ecosistema específico como son los humedales y ha permitido que, en este trabajo, se propongan estrategias, técnicas y factores que puedan ayudar a cambiar la percepción negativa existente y que faciliten la reconexión de la gente con los humedales.

\section{LA EXPERIENCIA TURÍSTICA EN HUMEDALES}

Para poder conseguir el aprecio del público por los humedales existen diversas oportunidades, pero quizá la más habitual e importante sea durante el momento mismo en que se visita el humedal. Es entonces cuando las autoridades gestoras responsables del lugar pueden poner en marcha estrategias, técnicas y programas in situ que se dirijan a cautivar y seducir al público. Por ello, en este apartado se analiza el proceso mental de construcción de una experiencia recreativa o turística, para así identificar en qué fases y de qué manera los gestores van a poder influir en el visitante para que se consiga el objetivo final: un comportamiento de aprecio por el humedal.

\subsection{Necesidades, motivaciones y expectativas previas a la visita a los humedales}

La experiencia recreativa propiamente dicha, como hemos comentado, tiene lugar in situ, durante la visita, pero realmente está constituida por una serie de procesos mentales que el visitante empieza a construir antes de la misma y también con otros procesos que pretendemos que perduren posteriormente a la visita. Así, tenemos que previamente a la visita, el individuo manifiesta unas necesidades, motivaciones y expectativas al respecto. El comportamiento es ya un resultado y se comienza a producir durante la visita pero se pretende que pase a formar parte del estilo de vida del visitante en un futuro.

Antes de seguir, hay que resaltar que el perfil del visitante y sus rasgos de personalidad influyen mucho en el desarrollo de este proceso. Por ello, procede identificar las principales tipologías de visitantes que frecuentan los humedales. Así, por una parte tenemos los observadores de aves, los grupos escolares, el público generalista y, finalmente, 
tenemos a un tipo de visitantes especializados que recientemente practican actividades deportivo-aventureras.

Los observadores de aves son visitantes bien conocidos en estos ecosistemas aunque el volumen que representan respecto al total es bajo. Se trata de una tipología de visitantes que ha sido ampliamente estudiada en trabajos sobre turismo en humedales (Kim et al. 1997; Hvenegaard, 2002; Scott y Thigpen, 2003; Conell, 2009; Carver, 2013) y no son el objeto principal de este estudio ya que estos visitantes encuentran en los humedales aquello que buscan y por tanto, se puede decir que sus necesidades quedan satisfechas. Además, están sensibilizados, aprecian los valores y funciones de los humedales y tienen actitudes y comportamientos proactivos de conservación.

Otro tipo de visitante también conocido en los humedales y representando un volumen importante, son los integrantes de grupos escolares que suelen realizar actividades interpretativas y/o educativas en estos ecosistemas y a los que se han dirigido todos los esfuerzos de sensibilización durante años. Se puede decir, en general, que es un público que aprecia los humedales pero que necesitan un constante refuerzo de los mensajes interpretativos, especialmente, los actitudinales y conductuales.

El mayor volumen de visitantes en los humedales lo constituye, sin embargo, el público general no especializado. Se trata de un grupo muy heterogéneo, cuyos rasgos de personalidad en términos generales, se definen por valores personales universales como la familia, amistad, bienestar, trabajo, etc. Las actitudes y comportamientos durante la visita, en ocasiones resultan inadecuados y difíciles de cambiar según se deriva de la experiencia propia en la puesta en marcha de programas de uso público en humedales. Son el tipo de público hacia el que este trabajo dirige su foco de atención ya que es el que necesita una mayor sensibilización y para el que resulta más difícil aprehender los valores y funciones de los humedales.

En años recientes, se observa también en los humedales la presencia de un tipo de visitante especializado y que lleva a cabo actividades deportivas y/o aventureras (si están permitidas en el lugar). Se trata de un grupo, entre cuyos valores personales destaca la aventura, la amistad, la salud, y el deporte. Son visitantes que no desprecian el conocimiento del humedal ni de sus valores pero su interés principal se centra en la actividad misma que van a desarrollar; el valor del humedal para ellos reside en la adecuación que presente para la práctica de su deporte, en el uso que hacen del humedal como escenario para su actividad, y es por ello que se les denomina natusers (Alonso-Monasterio et al., 2015).

Las necesidades recreativas que presentan cada uno de los tipos de visitantes mencionados son muy variables. En general, las necesidades recreativas basadas en la visita a humedales se relacionan con vínculos muy primarios que unen a las personas con la naturaleza, lo que se conoce bajo el concepto de Biofília (Wilson, 1986). Recientemente, autores como Louv (2005) identifican ciertos problemas en relación a este tema como el de la desconexión existente entre el hombre, especialmente los niños, y la naturaleza. Este autor sostiene que la falta de vínculos con la naturaleza puede ocasionar desórdenes físicos y psicológicos (Nature-Deficit Disorder), incluso es la causa de depresiones, e insiste en la necesidad de estimular que las nuevas generaciones de jóvenes desarrollen buenos recuerdos en relación a experiencias de recreación al aire libre. 
La recreación incluye toda una serie de actividades que el individuo lleva a cabo durante su tiempo libre y que satisfacen la necesidad de relajación y salud física y mental. En los humedales, los grupos con necesidades más específicas son los observadores de aves; siendo el público generalista el que tiene unas necesidades más básicas, pero no por ello menos importantes, y también siendo este público el que menos percibe esta necesidad. En la satisfacción de las necesidades, realmente el gestor del humedal puede influir poco, pues éstas dependen en gran medida de los rasgos de personalidad del individuo y de su entorno familiar y social. Los medios de comunicación, especialmente los de masas (TV, cine, etc.) a través de documentales, reportajes, etc. sí contribuyen a despertar ciertas necesidades de naturaleza en los individuos, incluso marcan tendencias y modas al respecto.

En cuanto a las motivaciones, hay que mencionar que se trata de las fuerzas internas que conducen a las personas a actuar para satisfacer las necesidades. Luo y Deng (2008) y Line y Costen (2011) han trabajado en la clasificación de las motivaciones de los visitantes de espacios naturales e incluyen básicamente cuatro tipos que, en nuestra opinión, resumen bien las propuestas en la literatura científica. De esta forma, tenemos que las cuatro motivaciones básicas por las que un individuo desea visitar la naturaleza son: 1 . una nueva vía de desarrollo personal; 2 . vuelta o reconexión con la naturaleza; 3. conocimiento y salud; y 4. escapada. Además, estos autores encuentran coincidencias positivas en las relaciones entre las actitudes medioambientales de los visitantes y su comportamiento durante la visita a espacios naturales. Se podría decir pues que todos los tipos de visitantes descritos tienen alguna de estas motivaciones para realizar la visita a los humedales. En cualquier caso, se puede señalar que el nivel de motivación dependerá también de las experiencias previas que han tenido en visitas similares. Por ejemplo, puede darse el caso de visitantes generalistas que llegan al humedal de forma casual, como parte de una excursión o tour mayor y, por tanto, lo hacen sin haber desarrollado ninguna motivación previa, en cuyo caso no se puede predecir cómo responderán ante la experiencia recreativa.

Las expectativas se generan a partir de las necesidades y las motivaciones, junto con la imagen del destino. Nuestra mente construye las expectativas a partir de la información que recibe sobre el lugar y crea una imagen proyectada. Echtner y Ritchie (1991) apuntaban que los individuos pueden tener una imagen de un destino incluso sin haberlo visitado o haber sido expuestos a fuentes de información comerciales, ya que las personas a lo largo de su vida van acumulando información relacionada con factores históricos, políticos, económicos y sociales que van conformando su imagen sobre un lugar en cuestión. La información que recibe un potencial visitante del humedal puede proceder de diversas fuentes, a las que Gartner (1993) denomina agentes de formación de la imagen, y Baloglu y McCleary (1999) llaman factores estímulo, y que son: las oficiales (en nuestro caso los gestores del humedal), comerciales (que serían los proveedores de servicios turísticos en la zona), y de otros prescriptores (como suelen ser amigos o familiares).

El gestor del humedal es la fuente oficial de información y su responsabilidad es suministrar de forma veraz toda la información sobre el sitio para que la imagen proyectada sea real. Una información errónea o sin actualizar puede acabar en una experiencia insatisfactoria si las expectativas del visitante no armonizan con la imagen del lugar. Este tema es especialmente relevante en el caso de los observadores de aves y visitantes especializados que necesitan encontrar los atractivos específicos por los cuales se han desplazado, ya que de otra manera se frustrarían sus expectativas, creando un gran 
disconfort psicológico difícil de restaurar posteriormente aunque existan otras oportunidades y atractivos en el lugar. Los grupos escolares pueden apreciar otros atractivos y quedar igualmente satisfechos, mientras que el público generalista va a ser el menos exigente en este tema y más si no habían albergado una motivación específica previa para la visita.

En relación a las fuentes comerciales, hay que comentar que son las más interesadas y parciales y por tanto, el visitante está más expuesto a tener una mala experiencia si construye su imagen proyectada a partir únicamente de esta información. Por el contrario, los prescriptores (por ejemplo otros usuarios que han visitado previamente el humedal) dan incluso una confianza mayor que la proporcionada por las fuentes oficiales. Así, además del boca-oreja o recomendación, que funciona bien entre el público generalista, hay que destacar en la difusión de esta información los documentales televisivos editados por productoras de prestigio como National Geographic, Lonely Planet, canal Viajar, Discovery Channel, etc. Hay que mencionar también el uso que hacen de las redes sociales (blogs, Facebook, Twitter, foros especializados, etc.) los observadores de aves y visitantes especializados, siendo éstos los principales canales de información para estos visitantes como se puede apreciar en Internet.

\subsection{La percepción del humedal y la experiencia recreativa}

La experiencia recreativa es el resultado de vivir determinadas situaciones, por tanto se produce in situ durante la visita al humedal, y es cuando se establece una conexión muy directa y personal entre éste y el visitante. La experiencia recreativa depende, en gran medida, del perfil del visitante, de sus rasgos de personalidad y de sus necesidades de naturaleza, motivaciones y expectativas, y se construye a partir de la percepción de los atractivos, de la actividad llevada a cabo y del confort con que se haya desarrollado todo el proceso. Todo ello se completa con la calidad del servicio y de los equipamientos, pasando entonces a poder considerarse como una experiencia turística propiamente.

De este modo tenemos que, una vez en el humedal, lo primero a lo que un visitante se enfrenta es su percepción del lugar, que es un proceso mediante el cual, según Mayo y Jarvis (1981), la persona recibe información del entorno a través de los sentidos, la selecciona, la organiza y la interpreta para crearse una imagen significativa o imagen percibida del sitio. Al ser un proceso que se realiza ya en el lugar y, para poder dar significado a la información que el individuo recibe, es necesaria una integración de la misma con elementos cognitivos previos (recuerdos, ideas, creencias, sentimientos previos, modelos, experiencias anteriores, etc.) que lógicamente varían según cada visitante tal como apuntan Pizam y Mansfel (1999) y Shiffman y Lazar (2000). Por tanto, vemos que en este proceso perceptivo interviene la memoria, el aprendizaje y la inteligencia tal como apuntaba Feldman (1999) cuando destacaba que esta actividad no sólo era tarea de los órganos sensoriales sino también del cerebro. Un elemento importante a considerar en la percepción (Pike y Ryan, 2005; Andrade, 2012), es además de la dimensión cognitiva o racional (conocimiento de los atributos: recursos, atractivos, equipamientos y servicios, etc.), la dimensión afectiva o emocional, que resulta especialmente interesante para la evaluar la satisfacción y para la sensibilización del público generalista. 
Los atractivos de los humedales son por tanto el objeto de aprehendizaje en el proceso de percepción. De esta forma, tenemos el paisaje, los componentes hidromorfológicos, la fauna, la flora, los elementos del patrimonio cultural y las propias comunidades locales y poblaciones indígenas en su caso; todos ellos van a ser objeto de atención de nuestro cerebro. Análisis científicos y años de experiencia en el desarrollo de programas de conservación y uso público en humedales basados en la aplicación de criterios de significancia, representación, singularidad, diversidad, naturalidad/integridad y rareza, nos informan del importante valor científico y patrimonial intrínseco que, desde el punto de vista geológico, geomorfológico, hidrológico, paleontológico, biológico, ecológico y también cultural (Viñals, 2002), detentan estos ecosistemas acuáticos, aunque éstos siguen resultando menos atractivos para el gran público que los de otros ecosistemas. Para acercarnos más al problema y conocer mejor el potencial de sus recursos para el uso público y, en particular, para desvelar la vocación interpretativa de los humedales procede pues aplicar otra serie de criterios como son: poder de atracción, fragilidad/vulnerabilidad, disponibilidad, accesibilidad, factibilidad, valores educacionales, aptitud para la recreación, capacidad de acogida y vocación para inducir a comportamientos de conservación y protección del patrimonio, más adecuados para describir la contribución a los procesos cognitivos descritos.

A este respecto hay que señalar que el poder de atracción de los recursos de los humedales puestos en valor hasta el momento ha resultado limitado para el público generalista. Así, salvo el caso de las aves, el resto de grupos faunísticos resulta: de tamaño diminuto (insectos), difíciles de observar (peces) o de difícil conexión emocional con el público (anfibios, reptiles); en el peor de los casos, cierta fauna es considerada molesta e incluso peligrosa. La vegetación casi nunca se ha puesto en valor en los programas de interpretación, aunque hay que decir que los paisajes sí han formado parte desde siempre del imaginario clásico de los humedales, ya que ofrecen cuencas visuales de gran belleza debido al efecto reflectante de las aguas. La biodiversidad, como atributo de mayor reconocimiento de un humedal, es un concepto que habitualmente está subestimado en el proceso de percepción. Los visitantes no suelen ser expertos taxónomos en biología o ecología y, por tanto, no son capaces de apreciar este atributo en su justa medida. Esto contrasta con la sensibilización mostrada por la megafauna que existe en otros ecosistemas que siempre resulta muy carismática (Kerley et al., 2003).

Una vez construida la percepción, ésta es evaluada por nuestra mente gracias a un proceso de comparación entre la imagen proyectada del lugar con la que se percibe finalmente; de ahí la importancia de hacer coincidir ambas imágenes tal como algunos autores como Kotler et al. (1993) sugieren. Esta evaluación se traduce en un juicio sobre si el rendimiento es: exactamente como se esperaba, mejor de lo que se esperaba, o peor de lo que se esperaba. En los dos primeros casos se producirá satisfacción, mientras que en el tercer caso se constatará claramente una insatisfacción. La satisfacción es un sentimiento de bienestar o placer que se tiene cuando se ha colmado un deseo o cubierto una necesidad. Este sentimiento es producto de la valoración cognitiva (Hunt 1975; Day, 1984; Fornell, 1992; Engel et al., 1993) y también emocional (Swan y Oliver, 1989; Oliver, 1993; Halstead et al., 1994; Spreng et al., 1996; Baker y Cromptom, 2000). Hay que procurar siempre que no se frustren las expectativas de los visitantes ya que, como Hsu et al. (2010) y Devesa et al. (2010) han demostrado, existen poderosos vínculos entre las motivaciones y expectativas de los turistas y sus comportamientos durante la visita. 
La reacción ligada al sentimiento de satisfacción, además de un estado de ánimo positivo en los visitantes, da lugar también a una actitud (cognitiva, emocional y conductual) y un comportamiento. Además de las actitudes positivas, determinados comportamientos son el fin último que persigue un gestor y son aquellos que pueden contribuir a la conservación del patrimonio y los recursos del humedal. No obstante, se puede ir más allá y plantearse objetivos más ambiciosos en relación a actitudes y comportamientos que puedan transcender al propio momento de la visita e influir en la vida cotidiana del visitante o en futuras visitas a otros humedales. Hay que mencionar que los sentimientos son más poderosos cuando se producen durante el momento de la visita, pero que pueden persistir en ausencia de los estímulos externos que los generaron, de ahí su valor para potenciar conductas posteriores en el visitante a medio y largo plazo.

Hay que resaltar además que el comportamiento del visitante in situ es una excelente herramienta para valorar la satisfacción (Lee, 2009; Prayag et al., 2013), el nivel de consecución de los objetivos planteados en los programas de interpretación y sensibilización, y para orientar las acciones de gestión (Bigné et al., 2001). Por tanto, nos podemos valer de la observación directa de determinadas actitudes y comportamientos y de la observación participante para evaluar la satisfacción del visitante. Investigadores como Faullant et al. (2008), Wang et al. (2009), Zabkar et al. (2010), Yuksel et al. (2010) y Rodger et al. (2012) han establecido que la satisfacción de los visitantes es un factor determinante de la fidelización al lugar, la recomendación del sitio a otros visitantes y también el apego personal al lugar (Folmer et al., 2013). Por ello, basándonos en estos trabajos, hay que pensar además que si un visitante ha podido completar el proceso de forma satisfactoria será un buen prescriptor del sitio.

\section{PROPUESTAS PARA LA MEJORA DE LA EXPERIENCIA RECREATIVA EN HUMEDALES}

Analizadas las dificultades que presentan los humedales para ser apreciados globalmente, se presentan en este apartado algunas propuestas, a modo de resultados, sobre estrategias, instrumentos para su puesta en valor y acciones a implementar para mejorar la percepción, las conexiones intelectuales y también las emocionales (fig. 1). 
Figura 1. Propuesta de acciones para mejorar la percepción de los humedales.

\begin{tabular}{|c|c|}
\hline $\begin{array}{l}\text { Acciones para la } \\
\text { mejora de la } \\
\text { percepción del } \\
\text { humedal }\end{array}$ & $\begin{array}{l}\text { - Mejora de la integridad/naturalidad y estado de conservación de } \\
\text { los recursos } \\
\text { - Refuerzos sensoriales para la potenciación de las sensaciones } \\
\text {-Prestación de un servicio de calidad (equipamientos, servicios, } \\
\text { atención al público, etc.) } \\
\text {-Provisión de confort psicológico al visitante (seguridad, } \\
\text { zonificación espacial, gestión de visitantes, patrón de la visita, } \\
\text { organización, tamaño y tipo de los grupos, espacio mínimo } \\
\text { personal, número de encuentros con otros grupos, etc.) }\end{array}$ \\
\hline $\begin{array}{l}\text { Acciones para la } \\
\text { mejora de las } \\
\text { conexiones } \\
\text { intelectuales } \\
\text { con el humedal }\end{array}$ & $\begin{array}{l}\text {-Disponer de un Programa de Interpretación que siga los principios } \\
\text { de Amenidad, Relevancia, Orden y focalizados en Temas o } \\
\text { mensajes principales } \\
\text { - Disponer de guías-interpretes bien formados en técnicas de } \\
\text { interpretación } \\
\text {-Utilización de iconos interpretativos que resulten familiares, } \\
\text { amigables y atractivos para el público generalista (mamíferos, } \\
\text { crías de animales, etc.) }\end{array}$ \\
\hline $\begin{array}{l}\text { Acciones para el } \\
\text { establecimiento } \\
\text { de conexiones } \\
\text { emocionales con } \\
\text { el humedal }\end{array}$ & $\begin{array}{l}\text { - Incorporación de mensajes o temas interpretativos con } \\
\text { significados que apelen a los conceptos o valores universales de } \\
\text { las audiencias } \\
\text { - Disponer de guías-interpretes bien formados en técnicas de } \\
\text { interpretación } \\
\text { - Incorporar en los programas el patrimonio cultural y el intangible } \\
\text { - Facilitar la conexión emocional con el pasado y las memorias } \\
\text { personales o familiares a partir de técnicas interpretativas como } \\
\text { la teatralización, performances, reconstrucciones históricas, etc. }\end{array}$ \\
\hline
\end{tabular}

\subsection{Acciones para la mejora de la percepción del humedal}

Diversas acciones pueden llevarse a cabo para mejorar o intensificar la percepción del humedal. Entre ellas se pueden citar: la mejora de la integridad/naturalidad y estado de conservación de los recursos del humedal, la potenciación de las sensaciones, la prestación de un servicio de calidad y la provisión del máximo confort psicológico posible al visitante. La primera tiene que ver con tareas de mantenimiento y restauración ecológica, mientras que las restantes tienen relación con la calidad del servicio.

La mejora de la percepción de un humedal debe plantearse desde la base de considerar el estado de conservación de los recursos, que son los principales atractivos del lugar y por tanto el elemento motivador más importante de la visita.

Otras técnicas que mejoran la percepción son los refuerzos sensoriales; es decir, hay que potenciar la utilización de todos los sentidos en el acto de aprehender la información. Los seres humanos basamos nuestra percepción sobre todo en la vista, quedando el resto de los sentidos infrautilizados; sentidos como el olfato tienen un gran poder evocador y el paisaje sonoro generado por las aves, por ejemplo, es un gran recurso que se puede utilizar en la puesta en valor de los humedales. 
La calidad del servicio es un elemento fundamental en la valoración de la satisfacción global de la visita y de la calidad de la experiencia tal como sugieren Tian-Cole y Crompton (2003). Este concepto hace referencia a los beneficios obtenidos en el lugar, incluyendo en la valoración elementos tales como la calidad de los equipamientos y servicios y la atención al público del personal (amabilidad, educación, preparación, profesionalidad, etc.). Hay que recordar que estos elementos son susceptibles de ser gestionados y, por tanto, mejorados. La existencia de más o menos equipamientos no es sinónimo de calidad, pero hay dos premisas que se deben tener en cuenta: que unos equipamientos básicos (acogida e información, aseos, señalización, aparcamientos y senderos) son necesarios incluso para acoger a los visitantes más conservacionistas; y la segunda es que los equipamientos deben estar en perfectas condiciones de mantenimiento ya que de otra forma podrían incluso causar inseguridad en el visitante. Otros equipamientos deseables para facilitar la visita son los interpretativo-educativos (rutas interpretativas y centros de interpretación) ya que están en la base del acceso intelectual al patrimonio. La señalización también es fundamental ya que contribuye a que los visitantes tengan seguridad e información, y son un vehículo importante del programa de interpretación, dan a conocer las pautas de comportamiento esperadas, y además dan orientación para seguir el patrón de la visita. En relación a los servicios, hay que señalar que son valorados dependiendo del perfil del visitante y de la calidad del servicio ofrecido, entendiendo ésta como el cumplimiento de la expectativa o la necesidad del visitante. El público más generalista necesita habitualmente más equipamientos y servicios; mientras que los visitantes especializados suelen obviarlos, e incluso pueden llegar a valorar negativamente su sobredimensionamiento. Los servicios interpretativos personales deberían ser un elemento irrenunciable que merece el público de cualquier humedal y que si se presta en condiciones apropiadas y con guías-intérpretes cualificados garantiza el acceso intelectual al patrimonio del lugar.

En relación al confort psicológico, hay que señalar que se asimila básicamente al de bienestar del individuo en relación al entorno donde se encuentra; es decir, es una expresión del equilibrio positivo entre el hombre y el medio e incluye varios factores a considerar (Viñals et al., 2014) y que influyen notablemente en la percepción del visitante; hay que garantizar que la experiencia recreativa discurra en términos de confort para que el visitante pueda absorber toda la información que el entorno le facilita y poder construir la percepción que se persigue procurando que nada ni nadie interfiera negativamente en este proceso. Los factores de confort psicológico sobre los que pueden influir los gestores para mejorar la percepción son en los relativos a proporcionar las máximas condiciones de seguridad y en los factores organizativos.

La gestión de las cuestiones de seguridad en los humedales es compleja, ya que en estos ecosistemas existen zonas con peligros reales para los visitantes. Así, además de espacios intransitables muy densamente vegetados, existen suelos inestables debido a la presencia de fangos o arenas movedizas que son incapaces de soportar la presión que ejerce el peso de una persona y, por tanto, son áreas donde los visitantes podrían tener problemas. Tampoco sus aguas suelen ser vadeables por el carácter fangoso de sus suelos, por lo que no son aptas para la gran mayoría de actividades acuáticas (caso de que fueran permitidas), ya que el acceso desde las orillas suele ser complejo e inseguro. De esta forma, vemos que es absolutamente necesaria la existencia de una zonificación debidamente señalizada del humedal donde se prohíba e impida el paso a los visitantes a ciertas áreas por cuestiones 
estrictas de seguridad. También es imprescindible que el humedal esté dotado de un programa de seguridad y emergencias y, sobre todo, que los usuarios estén informados, desde el principio, de los posibles riesgos y/o limitaciones existentes para la práctica de las actividades recreativas. Los usuarios deben visitar el lugar y desarrollar sus actividades en condiciones de seguridad y, además, deben percibirlo así para su tranquilidad. Por ello, todos estos términos deben estar claramente expuestos en forma de recomendaciones en la señalización, en los mapas, folletos y comunicarse a través de los informadores y guíasintérpretes. Un visitante bien informado acerca de las vicisitudes del lugar, realiza la actividad de forma más tranquila, confortable y relajada porque sabe con lo que se va a encontrar y cómo comportarse ante cualquier eventualidad.

Los factores organizativos están relacionados básicamente con la gestión de visitantes (flujos, saturación, congestión) que está muy ligado al patrón de la visita, la zonificación del área de uso público y la organización de los grupos. Respecto a los flujos hay que señalar en primer lugar, que se necesita saber el volumen aproximado de visitantes que el humedal recibe anualmente y su distribución a lo largo del año para poder planificar la gestión de los visitantes durante las diversas estaciones del año en función de las diferentes situaciones ecológicas del humedal (periodo de apareamiento, cría, migración, floración, sequía, inundación, etc.). El volumen global de frecuentación del humedal debe ser el adecuado para garantizar la conservación y protección de los recursos y aquel con el que los usuarios se sientan cómodos. Los humedales no suelen presentar saturación generalizada pero sí cierta congestión puntual en determinados lugares o equipamientos, especialmente en los observatorios de aves y en ciertos senderos si no existe un patrón de la visita circular. La literatura científica turística ha dedicado muchos trabajos a este tema (Manning, 1999; Morgan y Lok, 2000; Vaske y Donnelly, 2002; World Tourism Organization, 2004) haciendo alusión a la insatisfacción de la experiencia recreativa que la saturación y congestión llevan aparejada a causa del disconfort psicológico que produce en aquellos visitantes que buscan especialmente ambientes donde disfrutar la naturaleza en soledad, como es el caso de los observadores de aves. En este sentido, hay que recordar que se debe dotar al humedal de herramientas preventivas como es la capacidad de carga recreativa para evitar estos problemas (Viñals et al., 2004).

En relación a la provisión de confort psicológico en la práctica de actividades recreativas, hay que comentar que, independientemente de qué tipo de actividad se practique (lúdica o de entretenimiento, interpretativo-educativa y/o deportivo-aventurera), lo importante es que el visitante disponga del espacio personal necesario para desarrollarla. En este sentido los estudios de Proxémica (Hall, 1963) son muy ilustrativos. Como se ha comentado, no es habitual que se produzcan condiciones de saturación y congestión en los humedales, por lo que el estándar de espacio personal suele estar garantizado incluso para aquellas actividades dinámicas que necesitan más superficie para su práctica.

El patrón de la visita y el tamaño y tipo de los grupos que se establece ayudan a evitar impactos en los recursos que se desea conservar y también facilitan el desarrollo de los programas de uso público. Un buen patrón de la visita, además de contribuir a la seguridad del visitante, permite un acceso tanto al medio físico en buenas condiciones (evitando la congestión y el encuentro con otros grupos, pasar dos veces por el mismo sitio, etc.), como intelectual (conocimiento de los valores patrimoniales del lugar) ya que se presentan los recursos de forma ordenada, secuencial o espacialmente, de forma que el 
visitante rápidamente pueda asimilarlos sin dificultad. De esta manera, se facilita una pauta para que el visitante no distraiga la atención en cómo evitar peligros y/o en cómo organizar y aprehender la información que se le está facilitando, y pueda por tanto disfrutar de una experiencia sensorial añadida.

En cuanto a la organización de los grupos hay que tener en cuenta: el tamaño, perfil de los componentes del grupo y los encuentros con otros grupos. En términos generales, se puede decir que cuanto más pequeño es el grupo, mayor confort se puede proveer ya que se pueden personalizar mejor las acciones, y los programas de interpretación pueden funcionar mejor (Moscardo, 1996). Existen muchos estudios empíricos en espacios naturales donde se sugiere que nunca se debe exceder un volumen de diez integrantes. También se puede comentar que cuanto más afines sean los componentes de un grupo, mayor confort se constata porque todos ellos comparten motivaciones y expectativas similares y la eficiencia comunicativa va a ser mayor. Existen algunas tipologías de grupos, sobre todo los muy especializados, que son muy exigentes a este respecto y no se encuentran confortables sino es en compañía de otras personas con su mismo perfil y motivaciones, y en grupos de tamaño muy reducido; tal es el caso de los observadores de aves (Carver, 2013) a los que ya se ha aludido. Los visitantes de carácter más generalista admiten mejor las actividades en grupo y, además es más habitual, aunque desarrollan conjuntamente una actividad breve, que adquieran comportamientos grupales. De esta forma, a la hora de considerar el confort psicológico de un grupo se tendrá en cuenta que, en el primer caso, necesitan un tratamiento de conjunto ya que forman un todo y la satisfacción de sus integrantes dependerá no sólo del éxito del desarrollo de la actividad recreativa sino también de las interrelaciones de dependencia de sus miembros. De estos hechos se deriva la importancia de saber componer grupos homogéneos con expectativas similares y de reconocer cuando hay una mala situación grupal. Como se puede intuir, organizar grupos homogéneos no resulta fácil si no se tiene información previa sobre sus componentes. En cuanto al número de encuentros, los estudios de McCool (1983) establecen que encontrarse con más de diez grupos a lo largo de una experiencia recreativa es demasiado y, por tanto podría contribuir al disconfort psicológico del visitante.

\subsection{Acciones para la mejora de las conexiones intelectuales con el humedal}

La primera propuesta para poder establecer y/o mejorar las conexiones intelectuales entre las personas y un humedal es la de disponer de un Programa de Interpretación ya que son una herramienta fundamental para la transmisión eficiente de conocimiento. Los programas, tal como sugiere Ham (1993), tienen que caracterizarse por ser amenos, relevantes, organizados y temáticos. El énfasis de este autor recae sobre el enfoque temático; es decir, se trata programas de interpretación que deben basarse en temas o ideas centrales (entre 3 y 5 ) para guiar el desarrollo del proceso de comunicación.

Los programas de interpretación se plasman tanto en los paneles utilizados en rutas o centros de interpretación como en el relato de los guías. Se trata de documentos técnicos que incluyen técnicas específicas (Ham, 1993; 2013; Veverka, 2002) para poder dar un significado a la nueva información que recibe el visitante sobre el humedal. Los mejores resultados en el desarrollo de programas de interpretación se obtienen con los guíasintérpretes porque permiten una mayor y más directa interacción con el visitante y una mejor adaptación del programa a sus necesidades. 
A pesar de todo lo expuesto, puede darse el caso que la información proporcionada no resulte interesante para el público generalista, por lo que otra estrategia y acciones deberán implementarse como es la identificación y utilización de nuevos iconos interpretativos de los humedales. De esta forma, se facilita la creación de imaginarios que constituyan atractivos más sencillos de interpretar y tengan un significado para este público. Se considera importante incluir entre los nuevos iconos a mamíferos y otros grupos faunísticos y florísticos que resulten más familiares, amigables y atractivos para el público generalista como: los perros de agua, caballos, ciervos, castores, nutrias, capibaras, manatíes, macacos, etc. y toda la innumerable variedad de nenúfares y flores de loto (Nymphaeaceae, Nelumbonaceae, Cabombaceae) o los micrófitos flotantes del género Eichhormia (lirios acuáticos, camalotes, jacintos de agua, etc.) que embellecen la superficie de los humedales. Las especies faunísticas mencionadas no suelen ser las abanderadas o flagship species de los humedales pero resultan eficientes a la hora de conseguir los objetivos de conservación y aprecio del humedal por parte del público generalista. Kerley et al. (2003) las denominan "especies paraguas" (umbrella species) y argumentan que para que los visitantes puedan experimentar la biodiversidad en toda su plenitud y apoyen la conservación del sitio, es suficiente con la observación de estas especies. Leader-Williams y Dublin (2000) abundan en esta idea y apuntan a que las especies grandes y carismáticas atraen la atención del público tanto como las especies abanderadas. La inclusión de mamíferos en este nuevo imaginario se justifica debido a la atracción que muestran en general los visitantes por esta clase de vertebrados. Así, estudios llevados a cabo sobre las preferencias de los visitantes en relación a la fauna, efectuados fundamentalmente en parques zoológicos (Bitgood et al., 1988; Reade y Waran, 1996; Balmford et al., 1996; Ward et al., 1998; Balmford, 2000; Moncada et al., 2002; Margulis et al., 2003; Véras dos Santos et al., 2012) confirman estos resultados. El hecho de incluir entre los nuevos iconos también a animales de compañía o mascotas tiene que ver con la cercanía y empatía que tienen con las personas, facilitando así la conexión intelectual y también la emocional. No hay que olvidar que entre los animales de compañía más populares se encuentran especies típicas de humedales como los perros, caballos, roedores, aves, algunos reptiles (tortugas) y mascotas acuáticas como peces y ranas.

\subsection{Acciones para el establecimiento de conexiones emocionales con el humedal}

En relación al proceso emocional, hay que comentar que la generación de emociones es un elemento fundamental para poder garantizar al visitante una experiencia turística satisfactoria. De cualquier manera, hay que señalar que la conexión emocional que se busca va más allá de la satisfacción o el placer de la visita o de los productos o servicios recibidos, implica la búsqueda de sentimientos de felicidad, conexión con la naturaleza o la historia, orgullo, etc. y así crear un verdadero vínculo personal con el lugar. Algunos autores opinan que la generación de emociones debería considerarse incluso como un fin en sí mismo.

De esta forma, cada vez con más fuerza, se apunta hacia la consecución de emociones durante el transcurso de la visita ya que los vínculos emocionales con el humedal predisponen a la acción y contribuyen decisivamente a generar sentimientos positivos de satisfacción que, a su vez, dan lugar a actitudes de aprecio que conducen hacia la conservación y el disfrute del espacio natural. Habitualmente, los programas de interpretación suelen centrarse en el campo del conocimiento cognitivo y son pocos los que han desarrollado programas en donde se incorpora la dimensión emocional. Sería pues 
interesante que se reforzaran los programas de interpretación incorporando elementos que facilitaran el desarrollo de vivencias y emociones.

Entre las estrategias que se sugieren para mejorar las conexiones emocionales entre los visitantes y el humedal destaca la inclusión de mensajes o temas interpretativos con significados que apelen a los valores personales de las audiencias. Estos mensajes deben ser veraces pero, a su vez, deben incorporar una componente personal con rasgos de compromiso basados en los valores o conceptos universales que todas las personas tienen. Estos valores son los que confieren relevancia al mensaje; de esta manera, se observa que mensajes ligados a los valores humanos pueden desencadenar las emociones de los visitantes. Un ejemplo de esta reflexión es el hecho de que la observación de las crías de los animales resulta emocionalmente eficiente ya que aproxima o vincula al individuo con la fauna al aludir al instinto protector común que personas y animales comparten.

Otra estrategia para generar emociones durante la visita es la utilización de guíasintérpretes. La comunicación personal siempre resulta más atrayente que la facilitada por recursos no personales (paneles, videos, folletos, etc.). Las razones para ello se basan en que muchos visitantes no son capaces por sí solos de conectar en toda su dimensión con los conocimientos y estímulos que envía la naturaleza ya que, como se ha comentado, resultan ecosistemas difíciles y además muchas audiencias están desconectadas de la naturaleza por el estilo de vida urbano imperante. De este modo, un guía-intérprete puede ayudar en esta tarea de reconexión. La segunda razón, y además muy poderosa, es que los seres vivos y, en particular los humanos, son los más capaces de generar emociones a otro ser humano. Se deriva obviamente de estos comentarios, la importancia que tiene la formación del personal encargado de este cometido. En muchas ocasiones, el éxito de la visita está en las manos de los guías-intérpretes, que además pueden ser capaces de resolver positivamente situaciones aún en condiciones externas de confort adversas.

Además de las estrategias y acciones presentadas, hay que considerar que el humedal será más significativo para los visitantes si, además de los elementos tangibles, logramos ligarlos a los significados intangibles del sitio. El patrimonio intangible es un elemento sustancial e integrador espacial de los paisajes culturales, con un gran poder generador de relaciones y vínculos entre las personas (cohesión social) y entre éstas y el humedal (poder de reconectar el hombre con la naturaleza), ya que este patrimonio suele estar ligado a las tradiciones locales $y$, en definitiva, a las personas, por lo que puede presentar una gran carga simbólica, valor testimonial, identidad colectiva y memoria histórica de una sociedad con la que el visitante podría identificarse. Un recurso importante en este caso es utilizar la teatralización, performances, reconstrucciones históricas, etc. para facilitar la conexión emocional con el pasado y las memorias personales o familiares y así crear vínculos con el lugar.

\section{CONCLUSIONES}

Como reflexiones finales se puede apuntar que la conservación de los humedales no está garantizada solamente con programas técnicos y figuras legales de protección, sino que la dimensión social de la conservación es tanto o más importante y es más garantista. Por ello, es necesario el desarrollo e implementación de instrumentos y herramientas técnicas 
de comunicación que acerquen estos ecosistemas al público, especialmente al público generalista que es el mayoritario y el más desatendido hasta el momento.

En este trabajo se ha expuesto una visión novedosa desde la que abordar programas de interpretación y campañas de sensibilización para humedales partiendo del análisis del proceso mental de construcción de necesidades, motivaciones, expectativas, percepción y satisfacción sobre los que se genera la experiencia recreativa en el humedal basada en el conocimiento pero, sobre todo, en las emociones.

Los resultados obtenidos apuntan hacia estrategias y técnicas que los gestores de humedales pueden aplicar en el proceso cognitivo y emocional que los visitantes desarrollan previamente a la visita a un humedal y, sobre todo, durante la misma, de manera que se facilite a los visitantes la consecución de una experiencia de calidad y satisfactoria en el lugar $y$, si es posible, que desarrollen emociones y sentimientos positivos que les induzcan a generar actitudes y comportamientos cada vez más comprometidos con la conservación y protección de los humedales.

Se destaca en este trabajo, entre otros resultados, la necesidad de potenciar la dimensión emocional de la experiencia recreativa. Para ello, se han propuesto y justificado la utilización de diversas estrategias como: identificar e incorporar en los programas interpretativos nuevos iconos o atractivos más sencillos y familiares para que puedan ser fácilmente aprehendidos por el público generalista, así como el desarrollo de mensajes interpretativos más personalizados, potenciar los refuerzos sensoriales en la fase experiencial de la visita, la utilización de guías intérpretes cualificados como canal comunicativo prioritario y la necesidad de incorporar el patrimonio cultural y el intangible para reforzar las conexiones emocionales entre el visitante y el humedal a través de historias, memorias y recuerdos.

\section{REFERENCIAS BIBLIOGRÁFICAS}

Alonso-Monasterio, M., Alonso-Monasterio, P. y Viñals, M.J. (2015). Natusers' motivations and attitudes in urban green corridors: challenges and opportunities. Case study of the Parc Fluvial del Túria (Spain). Boletín de la Asociación de Geógrafos Españoles, 68, 369383.

Andrade, M.J. (2012). La interpretación de la realidad del destino por parte de los turistas: evaluaciones cognitivas y afectivas. PASOS. Revista de Turismo y Patrimonio Cultural, 10(5), 477-494.

Baker, D.A. y Crompton, J.L. (2000). Quality, satisfaction and behavioral intentions. Annals of Tourism Research, 27(3), 785-804.

Balmford, A. (2000). Separating fact from artifact in analyses of zoo visitor preferences. Conservation Biology, 14, 1193-1195.

Balmford, A., Mace, G.M. y Leader-Williams, N. (1996). Designing the ark: setting priorities for captive breeding. Conservation Biology, 10, 719-727.

Bernués, M., Custodio, E. Torán, T. y Viñals, M.J. (2001). Aguas subterráneas y humedales (ISBN 84-95516-36-5). Ed. Fundación Marcelino Botín. Col. Papeles del Proyecto Aguas Subterráneas, serie $C$, Madrid.

Bigné, J.E., Sánchez, M.I. y Sánchez, J. (2001). Tourism image, evaluation variables and after purchase behaviour: inter-relationship. Tourism Management, 22, 607-616. 
Bitgood, S., Patterson, D. y Benefield, A. (1988). Exhibit design and visitor behaviour: empirical relationships. Environment and Behaviour, 20, 474-491.

Baloglu, S. y McCleary, K.W. (1999). A model of destination image formation. Annals of Tourism Research, 26(4), 868-897.

Carver, E. (2013). Birding in the United States: A Demographic and economic Analysis. Addemdum to the 2011 National Survey of Fishing, Hunting, and Wildlife-Associated Recreation. U.S. Fish \& Wildlife Service, USA.

Conell, J. (2009). Birdwatching, Twitching and Tourism: towards an Australian perspective. Australian Geographer, 40(15), 203-217.

Day, R.L. (1984). Modeling Choices Among Alternative Responses to Dissatisfaction. Advances in Consumer Research, 11, 496-499.

Devesa, M., Laguna, M. y Palacios, A. (2010). The role of motivation in visitor satisfaction: Empirical evidence in rural tourism. Tourism Management, 31(4), 547-552.

Echtner, C.M. y Ritchie, J.R.B. (1991). The meaning and measurement of destination image. The Journal of Tourism Studies, 2(2), 2-12.

Engel, J.F.; Blackwell, R.D. y Miniard, P.W. (1993). Consumer Behaviour. Dryden Press.

Faullant, R., Matzler, K. y Fuller, J. (2008). The impact of satisfaction and image on loyalty: The case of alpine ski resorts. Managing Service Quality, 18(2), 163-178.

Feldman, R. (1999). Psicología. Mc Graw Hill (México D.F.).

Folmer, A., Haartsen, T. y Huigen, P.P.P. (2013). The role of wildlife in emotional attachment to a nature-based tourism destination. Journal of Ecotourism, 12(3), 131-145

Fornell, C. (1992). A national customer satisfaction barometer. The Swedish experience. Journal of Marketing, 56, 6-21.

Gartner, W.C. (1993). Image formation process. Journal of Travel and Tourism Marketing, 2(2-3), 191-215.

Hall, E.T. (1963): A system for the notation of Proxemic behavior. American Anthropogist, 65(1), 1003-1026.

Ham, S. (1993). Environmental Interpretation. A Practical Guide for People with Big Ideas and Small Budgets. Fulcrum Publishing, Colorado.

Ham, S. (2013): Interpretation: Making a Difference on Purpose. Fulcrum Publishing, Golden, Colorado.

Halstead, D., Hartman, D. y Schimdt, S.L. (1994). Multisource Effects on the Satisfaction Formation Process. Journal of the Academy of Marketing Science, 22, 114-129.

Hsu, C.H.C., Cai, L.A. y Li, M. (2010). Expectation, motivation, and attitude: A tourist behavioral model. Journal of Travel Research, 49(3), 282-296.

Hunt, J.D. (1975). Image as a Factor in Tourism Development. Journal of Travel Research, 13, 1-7.

Hvenegaard, G.T. (2002). Birder specialization differences in conservation involvement, demographics, and motivations. Human Dimensions of Wildlife, 7, 21-36.

Kerley, G.I.H., Geach, B.G.S. y Vial, C. (2003). Jumbos or bust: do tourists' perceptions lead to an under-appreciation of biodiversity? SouthAfrican Journal of Wildlife Research, 33(1), 13-21.

Kim, S.S., Scott, D. y Crompton, J.L. (1997). An exploration of the relationships among social psychological involvement, behavioral involvement, commitment, and future intentions in the context of birdwatching. Journal of Leisure Research, 29(3), 320-341. 
Kotler, P., Haider, D.H. y Rein, Y. (1993). Marketing places: attracting investment, industry and tourism to cities, states and nations. The free Press, New York.

Leader-Williams, N. y Dublin, H.T. (2000). Charismatic megafauna as 'flagship species'. En A. Entwhistle \& N. Dunstone (Eds), Priorities for the conservation of mammalian diversity: has the panda had its day? (pp. 53-81). Conservation Biology Series, Cambridge University Press, Cambridge.

Lee, T.H. (2009). A Structural model to examine how destination image, attitude, and motivation affect the future behavior of tourists. Leisure Sciences, 31(3), 215-236.

Line, N. y Costen, W. (2011). Environmental attitudes, motivation, and attachment: Toward a model of nature-based tourism. International CHRIE, Paper 5.

Louv, R. (2005). Last child in the woods. Saving our Children from Nature Deficit Disorder. Algonquin Books of Chapel Hill.

Luo, Y. y Deng, J. (2008). The new environmental paradigm and nature-based tourism motivation. Journal of Travel Research, 46, 392-402.

Manning, R.E. (1999). Studies in outdoor recreation: Search and research for satisfaction. Oregon State University Press, Corvallis.

Margulis, S.W., Hoyos, C. y Anderson, M. (2003). Effect of felid activity on zoo visitor interest. Zoo Biology, 22, 587-599.

Martin, S.R. (1997). Specialization and differences in setting preferences among wildlife viewers. Human Dimension of Wildlife, 2, 1-18.

Mayo, E.J. y Jarvis, L.P. (1981). The psychology of the leisure travel: Effective marketing and selling of travel services. CBI Pub. Co., Boston, Mass.

McCool, S.F. (1983). The National Parks in Post-Industrial America. Western Wildlands, 9(2), 14-19.

Moncada, J. A., Aranguren, J., Díaz, E., del Castillo, M., y Benaya, J. (2002). Implicaciones prácticas de las preferencias de los visitantes del Parque Zoológico Caricuao. Caracas. Investigación y Postgrado, 17(1). 135-158.

Morgan, D.J. y Lok, L. (2000). Assessment of a Comfort Indicator for Natural Tourist Attractions: The Case of Visitors to Hanging Rock, Victoria. Journal of Sustainable Tourism, 8(5), 393-408.

Moscardo, G. (1996). Mindful Visitors: Heritage and Tourism. Annals of Tourism Research, 23(2), 376-397.

Oliver, R.L. (1993). Cognitive, Affective, and Attribute Bases of the Satisfaction Response. Journal of Consumer Research, 20, 418-430.

Pike, S. y Ryan, C. (2005). Destination positioning analysis through a comparison of cognitive, affective and conative perceptions. Journal of Travel Research, 42(4), 333-342.

Pizam, A. y Mansfeld, Y. (Eds.). (1999). Consumer Behavior in Travel and Tourism. The Haworth Hospitality Press, New York.

Prayag, G., Hosany, S. y Odeh, K. (2013). The role of tourists' emotional experiences and satisfaction in understanding behavioral intentions. Journal of Destination Marketing \& Management, 2, 118-127.

Reade, L.S. y Waran, N.K. (1996). The modern zoo: how do people perceive zoo animals? Applied Animal Behaviour Science, 47, 109-118

Rodger, K., Moore, S.A. y Taplin, R. (2012). Visitor satisfaction, loyalty and protected areas: a review and the future. Informe WA Department of Environment and Conservation, Parks Victoria and the Parks Forum. Technical Report, Murdoch University. 
Scott, D. y Thigpen, J. (2003). Understanding the Birder as Tourist: Segmenting Visitors to the Texas Hummer/Bird Celebration. Human Dimensions of Wildlife, 8, 199-218.

Shiffmann, L.G. y Lazar Kanuk, L. (2000). Consumer Behavior. Prentice Hall.

Spreng, R.A., MacKenzie, S.B. y Olsshvsky, R.W. 1996. A re-examination of the determinants of consumer satisfaction. Journal of Marketing, 60, 15-32.

Swan, J.E. y Oliver, R.L. (1989). Postpurchase communications by consumers. Journal of Retailing, 65(2), 516-33.

Tian-Cole, S. y Crompton, J.L. (2003). A conceptualization of the relationships between service quality and visitor satisfaction, and their links to destination selection. Leisure Studies, 22(1), 65-80.

Tremblay, P. (2002). Tourism wildlife icons: Attractions or marketing symbols? [online]. En CAUTHE 2002: Tourism and Hospitality on the Edge (624 - 638). Lismore, N.S.W. Cowan Universty Press.

Vaske, J.J. y Donnelly, M.P. (2002). Generalizing the encounter-norm-crowding relationship. Leisure Sciences, 24, 255-269.

Véras dos Santos, E., De Souza, I., Guedes, D. y Biccas-Marques, J.C. (2012). What makes a mammal attractive to the public at the Zoological park of Sapucaia du Sul in Southern Brazil? Zoo's Print, XXVII (2), 5-11.

Veverka, J.A. (2002). Planning for Interpretative Training Courses. Interpscan 28(3).

Viñals, M.J. (Ed.). (2002). El patrimonio cultural de los Humedales / Wetland Cultural Heritage. Ministerio de Medio Ambiente de España.

Viñals, M.J., Morant, M., Hernández, C., Ferrer, C., Quintana, R. D., Maravall, N., Cabrelles, G., Ramis, J. y Bachiller, C. (2004). Albufera de Valencia (Spain): Measuring carrying capacity in a fragile ecosystem. En Indicators of sustainable development for tourism destinations: A Guidebook (pp. 330-337). World Tourism Organization, Madrid.

Viñals, M.J., Morant, M. y Teruel, M.D. (2014). Confort psicológico y experiencia turística. Casos de estudio de espacios naturales protegidos de la Comunidad Valenciana (España). Boletín de la Asociación de Geógrafos Españoles, 65, 293-316.

Wang, X., Zhange, J., Gu, C. y Zhen, F. (2009). Examining antecedents and consequences of tourist satisfaction: A structural modeling approach. Tsinghua Science and Technology, 14(3), 397-406.

Ward, P.I., Mosberger, N., Kistler, C. y Ficher, O. (1998). The relationship between popularity and body size in zoo animals. Conservation Biology, 12, 1408-1411.

Wilson, E.O. (1986). Biophilia. Harvard University Press.

World Tourism Organization (2004). Tourism Congestion Management at Natural and Cultural Sites. UNTWO, Madrid.

Yuksel, A., Yuksel, F. y Bilim, Y. (2010). Destination attachment: Effects on customer satisfaction and cognitive, affective and conative loyalty. Tourism Management, 31, 274284.

Zabkar, V., Brencic, M. y Dmitrovic, T. (2010). Modelling perceived quality, visitor satisfaction and behavioural intentions at the destination level. Tourism Management, 31, 537-546.

Para citar este artículo: Viñals, M. J. y Alonso, P. (2016). Técnicas y estrategias para desarrollar el turismo experiencial en humedales. Investigaciones Turísticas, (12), 1-22. http://dx.doi.org/10.14198/INTURI2016.12.01 